[Chem. Pharm. Bull.]

36( 3 ) $988-993(1988)]$

\title{
Rapid Characterization of Natural and Biotechnologically Synthesized Human Growth Hormones by Fast Atom Bombardment Mass Spectrometry and High-Performance Liquid Chromatography
}

\author{
Hiroshi NaKaZawa \\ Takarazuka Research Center, Sumitomo Chemical Co., Ltd., \\ 4-2-1, Takatsukasa, Takarazuka, Hyogo 665, Japan
}

(Received August 3, 1987)

\begin{abstract}
Fast atom bombardment mass spectrometric (FAB-MS) analysis of tryptic digest of human growth hormone $(\mathrm{hGH})$ was carried out to verify the primary structures of two kinds of hGHs expressed in Escherichia coli; native type $(\mathrm{r}-\mathrm{hGH})$ and $\mathrm{N}$-terminal methionine-containing type of hGH (met-hGH). Both structures were confirmed by comparing the mass spectra of the tryptic digest mixtures with that of authentic hGH extracted from human pituitary (p-hGH). The signals detected in the mass spectra accounted for $82 \%$ of the sequence of hGH. In order to determine the location of disulfide bonds, a method was designed for selective preparation of peptide fragments containing a disulfide bond from the tryptic digest. The selection was performed by comparison with high-performance liquid chromatograms before and after the reduction of the digest mixtures with dithiothreitol (DTT). From the mass spectra of two disulfide-containing peptide fragments, the locations of two disulfide bonds in the hGH sequence were confirmed to be the same among the three types of hGHs. The present study indicates that the FAB-MS provides an excellent method for the rapid verification of the primary structure of proteins, and the combination of highperformance liquid chromatography (HPLC) with this method facilitates the analysis of disulfide bonds.
\end{abstract}

Keywords-human growth hormone; FAB-MS; HPLC; protein structure; disulfide bond; tryptic digest; peptide

\section{Introduction}

Human growth hormone ( $\mathrm{hGH}$ ) is a protein consisting of 191 amino acids, ${ }^{1,2)}$ which is secreted from the anterior lobe of the pituitary, and is an important therapeutic agent for dwarfism. Recently, bacterial production of hGH has been established by the use of recombinant deoxyribonucleic acid (DNA) techniques. ${ }^{3,4)}$ The recombinant DNA method gives $\mathrm{N}$-terminal methionine-containing type of hGH as well as the native one. In this paper, the author defines hGH originating from human cadavers as " $\mathrm{p}$-hGH", that from bacterial production as " $\mathrm{r}$-hGH" and the methionine-containing type as "met-hGH". Analyses of the primary structures of these hGHs have been carried out by using conventional methods. ${ }^{5,6)}$

These conventional methods include peptide mapping of the proteolytic digest of proteins by high-performance liquid chromatography (HPLC), amino acid analysis and sequence analysis. In these analyses, the proteolytic digestion is not always reproducible, and each peptide component in the proteolytic digestion mixture should be purified to homogeneity, and all peaks detected by HPLC should be analyzed.

Recently, Shimonishi et al. demonstrated that sequence identification of proteins can successfully be performed by field desorption mass spectrometry (FD-MS) or fast atom bombardment mass spectrometry (FAB-MS). These mass spectrometric methods have been developed with small amounts of protein enzymatic digests without separating the individual 
fragment peptides. ${ }^{7-9)}$

By using these techniques, Yazdanparast et al. also reported a method for detection and assignment of disulfide bonds in peptides. ${ }^{10,11)}$ However, this method requires signals due to a disulfide bond to be observable in the spectra. Thus, an alternative method is still desirable.

In this paper, the author wishes to report the application of FAB-MS to tryptic digest mixtures of hGHs and the combination of HPLC with FAB-MS for the rapid and simple identification of the primary structures of $\mathrm{r}-\mathrm{hGH}$ and met-hGH. More than $82 \%$ of the amino acid sequence could be identified by FAB-MS. Additionally, since signals due to disulfide bonds were not obtained in those spectra, a new and simple method was developed for assignment of disulfide bonds in peptides. This method includes a newly designed HPLC method for selective preparation of peptide fragments containing disulfide bonds. Combination of this HPLC method with FAB-MS facilitated the determination of the locations of disulfide bonds in the sequence of hGH.

\section{Experimental}

Materials — - p-hGH, met-hGH and r-hGH were produced by Kabi Vitrum.(Stockholm, Sweden). The purity of these samples was confirmed by sodium dodecyl sulfate polyacrylamide gel electrophoresis. L-1-Tosylamino-2phenylethyl chloromethyl ketone (TPCK)-treated trypsin, acetonitrile, trifluoroacetic acid (TFA) and dithiothreitol (DTT) were purchased from Worthington (NJ, U.S.A.), Kanto Chemical (Tokyo, Japan), Peptide Institute (Osaka, Japan) and Wako Pure Chemical (Osaka, Japan), respectively. Deionized and distilled water was used. Other reagents were of analytical reagent grade.

Reversed-Phase (RP) HPLC__A liquid chromatograph (model LC-4A) (Shimadzu, Kyoto, Japan) equipped with a gradient elution module and a variable-wavelength detector (model SPD-2A) set at $230 \mathrm{~nm}$ was used. A RP silica gel column, YMC -Pack AM-303 $\left(\mathrm{C}_{18}, 10 \mathrm{~nm}, 5 \mu \mathrm{m}, 4.6 \times 250 \mathrm{~mm}\right.$ : Yamamura Chemical, Kyoto, Japan), was used. A mixture of water and acetonitrile containing $0.1 \%$ TFA was used as the mobile phase at a flow rate of $1.0 \mathrm{ml} / \mathrm{min}$. A linear gradient of acetonitrile from 5 to $65 \%$ in $60 \mathrm{~min}$ was applied after sample injection.

Tryptic Digestion - The three kinds of hGH were digested with TPCK-treated trypsin for $12 \mathrm{~h}$ at a substrateenzyme ratio of about $50: 1(\mathrm{w} / \mathrm{w})$ at $\mathrm{pH} 8.0$ and $37^{\circ} \mathrm{C}$.

Mass Spectral Measurement-FAB-MS were obtained with a JEOL HX-110 double-focusing mass spectrometer. Samples $(5-10 \mu \mathrm{g})$ were dissolved in $20 \%(\mathrm{v} / \mathrm{v})$ aqueous acetic acid $(2 \mu \mathrm{l})$, transferred onto the sample holder and mixed with glycerol $(0.5 \mu \mathrm{l})$ and $\alpha$-thioglycerol $(1 \mu \mathrm{l})$ on the plate. Then, the sample holder was inserted into the FAB-MS ion source. FAB-MS conditions were as follows: ion source, xenon atom beam at $7 \mathrm{keV}$ accelerating potential; spectral range, $m / z 300$ to $m / z 3500$; accelerating potential, $10 \mathrm{keV}$, resolving power, 4000 . Mass calibration was done using a mixture of CsI and $\mathrm{KI}(1: 2, \mathrm{w} / \mathrm{w})$ as a mass reference.

Reduction of hGH Tryptic Digest__ DTT solution $(0.5 \mathrm{mg} / 25 \mu \mathrm{l})$ was added to the hGH tryptic digest $(50 \mu \mathrm{g} /$ $25 \mu \mathrm{l})$, and the mixture was allowed to stand for $20 \mathrm{~min}$ at room temperature.

\section{Results}

As shown in Fig. 1a and 1b, the mass spectral patterns of the tryptic digests of p-hGH and $\mathrm{r}-\mathrm{hGH}$ were nearly identical to each other. However, there are some differences between the spectra of the tryptic digests of p-hGH and met-hGH. By comparing Fig. 1c with Fig. la or $1 \mathrm{~b}$, a signal at $m / z 930.5$ was assigned to the $\mathrm{N}$-terminal sequence of $\mathrm{p}$-hGH or $\mathrm{r}$-hGH from position 1 to 8 , and the difference between $\mathrm{m} / \mathrm{z} 1061.4$ and $\mathrm{m} / \mathrm{z} 930.5$, namely 131 , was identical to the mass number of methionine residue. Therefore, the signal at 1061.4 was assigned to the $\mathrm{N}$-terminal sequence of met-hGH from position 0 to 8 . Most of the main signals detected in Fig. 1a, $1 \mathrm{~b}$ and $1 \mathrm{c}$ could be assigned and the results are shown in Fig. 2, which covers $82 \%$ of the whole amino acid sequence of hGHs.

The chromatographic patterns of the tryptic digests of $\mathrm{p}-\mathrm{hGH}$ and $\mathrm{r}-\mathrm{hGH}$ were virtually superimposable. However, only one peak in the chromatogram of met-hGH was found to show a different retention time from that of $\mathrm{p}-\mathrm{hGH}$ or $\mathrm{r}$-hGH (peak $\mathrm{A}$ and $\mathrm{A}^{\prime}$ in Fig. 3). This peak was presumed to be derived from the $\mathrm{N}$-terminal peptide fragment. ${ }^{5,6)}$ 

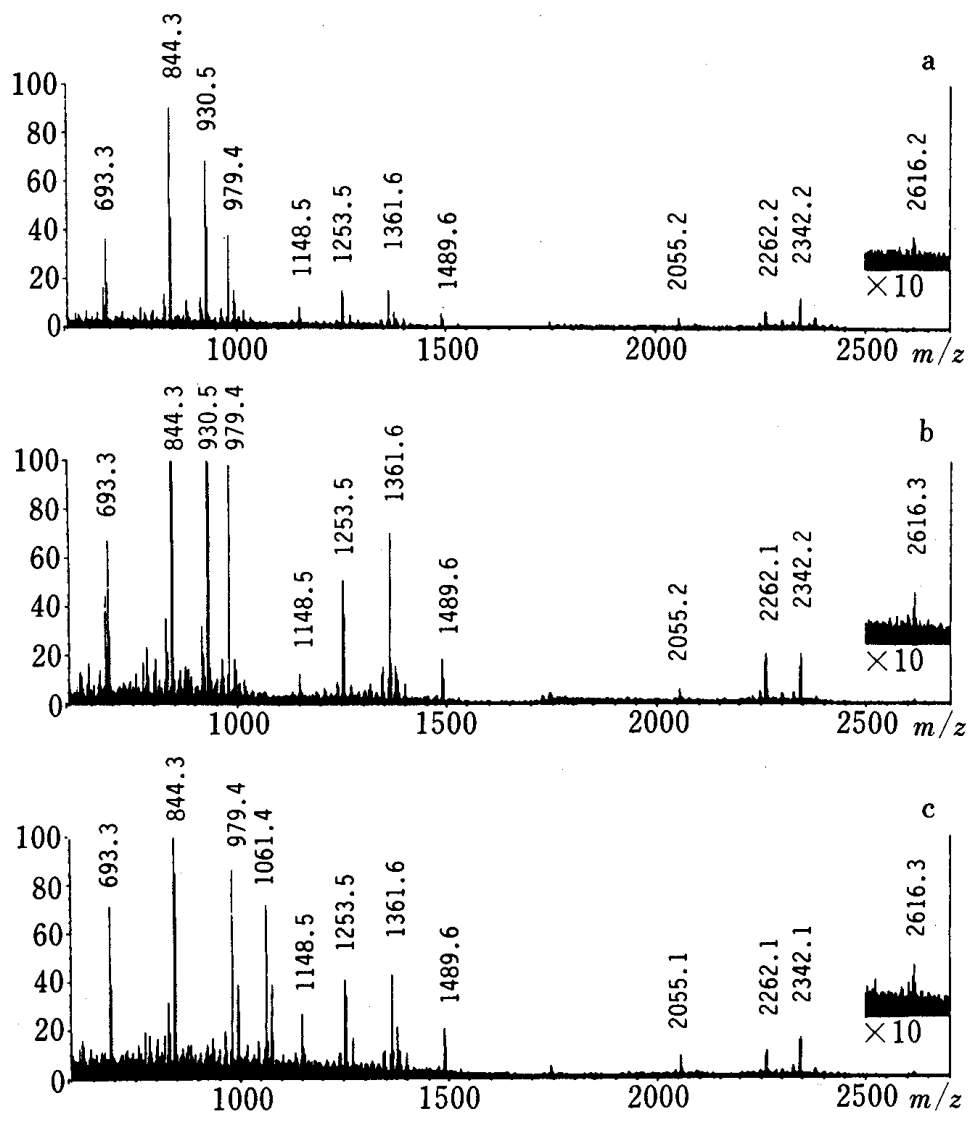

Fig. 1. FAB Mass Spectra of Tryptic Digests of hGHs

a, p-hGH; b, r-hGH; c, met-hGH. Numbers show observed mass values.

10

20

30

40

50

60

70

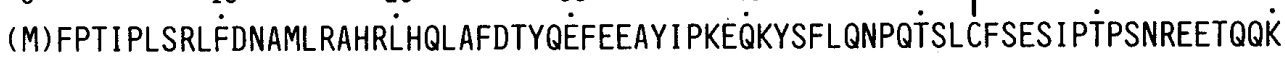

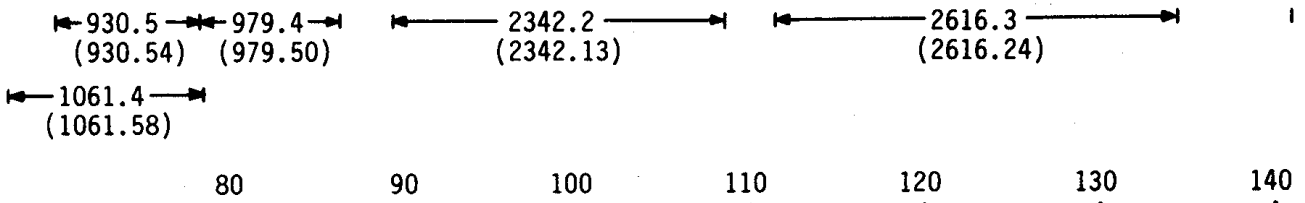

SNLELLRI SLLLLI QSWLEPVंQFLRSVFANS்LVYGASDSNVYYDLLKDLEEG I QTLMGRLEDGSPRTGQIFK

$1-844.3-4$

$(844.49)$

2055.2

$(2055.20)$

2262.1

$1361.6 \rightarrow$

$+693.3 \rightarrow$

(693.39)

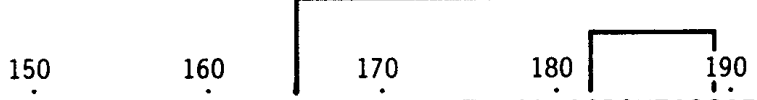

QTYSKFDTNSंHNDDALLKNYGLLYCFRKDMDDKVETFLRIVQCRSVEGSCं்

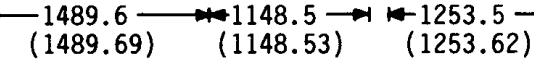

Fig. 2. Amino Acid Sequence of hGHs

Numbers without and with parentheses indicate observed mass values in Fig. 1a (p-hGH) and theoretical mass values, respectively. The signal at $m / z 1061.4$ in Fig. $1 \mathrm{c}$ was assigned to the $\mathrm{N}$-terminal sequence of residues $0-8$ in met-hGH, instead of the signal at $m / z 930.5$ which represents the sequence of residues $1-8$ in p-hGH or $\mathrm{r}$-hGH. The C-terminal sequence 179-191 was detected as shown in Fig. 4a. The abbreviations of amino acids used are according to IUPAC-IUB. ${ }^{12)}$ 


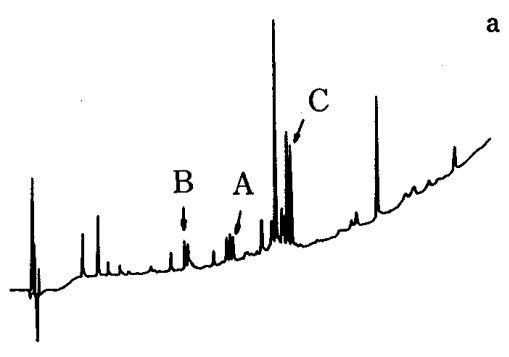

b
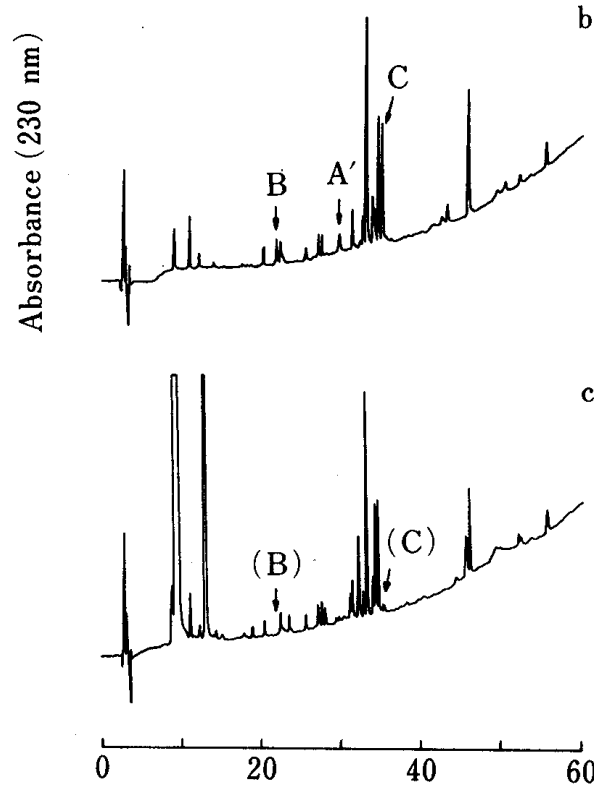

Retention time ( $\mathrm{min})$
Fig. 3. Reversed-Phase High-Performance Liquid Chromatograms of the Tryptic Digests of hGHs

a, p-hGH; b, met-hGH; c, tryptic digest of p-hGH reduced by dithiothreitol.

HPLC conditions are described in the experimental section.

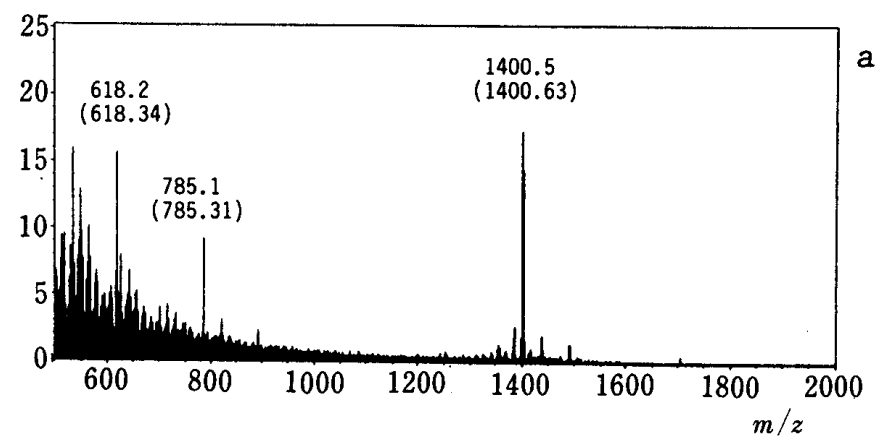

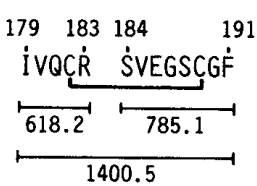

$\begin{array}{llll}42 & 64 & 159 & 167\end{array}$ YSFLQNPQTSLCFSESIPTPSNR NंYGLLYCFR

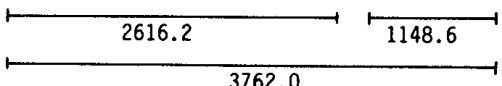

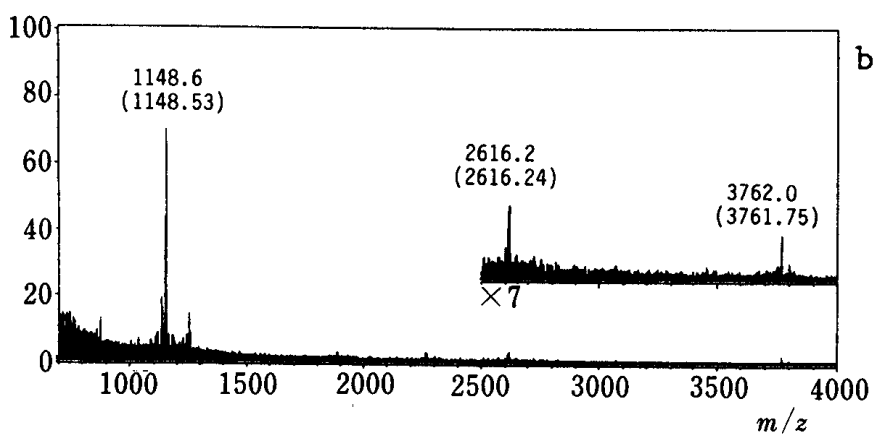

Fig. 4. FAB Mass Spectra of Tryptic Peptides of p-hGH Containing a Disulfide Bond and Their Signal Assignments

a, peak B; b, peak C (see the chromatogram of Fig. 3a). Numbers without and with parentheses indicate observed mass values and theoretical mass values, respectively. 
It was difficult to obtain information on disulfide bonds from the mass spectra of tryptic digests, since peptide fragments containing cysteine residue were detected as the signals of the reduced form. Therefore, individual peptide fragments which contain a disulfide bond in the tryptic digest of $\mathrm{p}-\mathrm{hGH}$ were fractionated by RP-HPLC, and subjected to mass spectrometric analysis. In order to detect selectively peptide peaks having a disufide bond among the peaks observed in Fig. 3, a small part of the tryptic digest of hGH was reduced by DTT treatment and the HPLC chromatograms obtained before and after the reduction were carefully compared. As shown in Fig. 3a and 3c, the peptides in peaks B and C in the chromatogram of Fig. 3a, which disappeared in the chromatogram of Fig. 3c, were found to contain a disulfide bond. These two peaks, fractionated by RP-HPLC, were subjected to mass spectrometric analysis. As shown in the mass spectrum of peak B (Fig. 4a), three signals at $m / z 618.2,785.1$ and 1400.5 were detected, which were assigned to the sequence at positions $179-183$ with Cys-182, 184-191 with Cys-189, and the sequence formed by the disulfide bonding of these two sequences, respectively. Similarly, the mass spectrum of peak $\mathrm{C}$ indicated a disulfide bond between Cys-53 and Cys-165 with the signals at 1148.6, 2616.2 and 3762.0 (Fig. 4b). These signals were assigned to the sequence $159-167$ with Cys-165, 41-64 with Cys-53, and the sequence formed by these two sequences with a disulfide bond. As a result, these mass spectra proved the presence of disulfide bonds between Cys-182 and Cys-189, and Cys-53 and Cys165. These results are consistent with the literature. ${ }^{5}$ In the same manner, two HPLC peaks in $\mathrm{r}-\mathrm{hGH}$ or met-hGH corresponding to peaks B and C in p-hGH were analyzed, and the same results were obtained. These results indicate that $\mathrm{r}-\mathrm{hGH}$ and met-hGH have the same disulfide bonds as those of $\mathrm{p}-\mathrm{hGH}$.

\section{Discussion}

By using the mass spectrometric method, peptide mapping can be performed by comparing spectral patterns, and concurrently, structural information can be obtained from the spectra. In fact, the mass spectra of tryptic digest mixtures of p-hGH, met-hGH and rhGH revealed accurately that $\mathrm{r}-\mathrm{hGH}$ has the same primary structure as $\mathrm{p}-\mathrm{hGH}$ does, and that met-hGH has an additional methionine residue as compared with $\mathrm{p}-\mathrm{hGH}$. Moreover, the mass spectra could account for $82 \%$ of amino acid sequence of hGH.

In the case of $\mathrm{hGH}$, the mass spectra of the tryptic digest mixture did not give information of disulfide bonds. Therefore, as described above, a new and simple method was developed for assignments of disulfide bonds in peptides. As a result, the position of disulfide bonds in the sequence could be easily identified by the measurement of the mass spectra of peptide fragments containing a disulfide bond isolated by RP-HPLC. These spectra of p$\mathrm{hGH}, \mathrm{r}-\mathrm{hGH}$ or met-hGH showed that they have disulfide bonds at the same positions. In the present study, the fragments containing a disulfide bond out of these peptide fragments can easily be isolated by comparison of chromatograms before and after the reduction of the proteolytic digest mixtures with DTT.

Thus, FAB-MS provides an excellent method for the verification of the primary structures of proteins produced by recombinant DNA techniques. Furthermore, the combination of HPLC with FAB-MS facilitates the analysis of disulfide bonds.

These techniques are also effective for the analysis of primary structures of analogous peptides and proteins. Although conventional sequence analysis and amino acid analysis are necessary for unknown samples, molecular weight determination by FAB-MS will give useful information for structural analysis.

Acknowledgment The author thanks Professor Y. Shimonishi of Osaka University for his helpful discussions and criticism of the manuscript, and Dr. T. Takao for his cooperation and valuable advice. The author is also indebted to Mr. Masayuki Nagase for his excellent technical assistance. 


\section{References}

1) C. H. Li, "Hormonal Proteins and Peptides," Vol. 3, Academic Press, New York, 1975, pp. 1-40.

2) J. A. Martial, R. A. Hallewell, J. D. Baxter and H. M. Goodman, Science, 205, 602 (1979).

3) D. V. Goeddel, H. L. Heyneker, T. Hozumi, R. Arentzen, K. Itakura, D. G. Yansura, M. J. Ross, G. Miozzari, R. Crea and P. H. Seeburg, Nature (London), 281, 544 (1979).

4) G. L. Gray, J. S. Baldridge, K. S. McKeown, H. L. Heyneker and C. N. Chang, Gene, 39, 247 (1985).

5) W. J. Kohr, R. Keck and R. N. Harkins, Anal. Biochem., 122, 348 (1982).

6) H. M. Hsiung, N. G. Mayne and G. W. Becker, BIO/TECHNOLOG Y, 4, 991 (1986).

7) Y. Shimonishi, Y. M. Hong, T. Matsuo, I. Katakuse and H. Matsuda, Chem. Lett., 1369 (1979).

8) Y. Shimonishi, "Methods in Protein Sequence Analysis," ed. by M. Elzinga, Human Press, New Jersey, 1982, pp. $271-278$.

9) T. Takao, T. Hitouji, Y. Shimonishi, T. Tanabe, S. Inouye and M. Inouye, J. Biol. Chem., 259, 6105 (1984).

10) R. Yazdanparast, P. Andrews, D. L. Smith and J. E. Dixon, Anal. Biochem., 153, 348 (1986).

11) R. Yazdanparast, P. Andrews, D. L. Smith and J. E. Dixon, J. Biol. Chem., 262, 2507 (1987).

12) IUPAC-IUB Joint Commission on Biochemical Nomenclature, Eur. J. Biochem., 138, 9 (1984). 